\title{
Suicides during pregnancy and 1 year postpartum in Sweden, 1980-2007
}

Annika Esscher, Birgitta Essén, Eva Innala, Fotios C. Papadopoulos, Alkistis Skalkidou, Inger Sundström-Poromaa and Ulf Högberg

\section{Background}

Although the incidence of suicide among women who have given birth during the past 12 months is lower than that of women who have not given birth, suicide remains one of the most common causes of death during the year following delivery in high-income countries, such as Sweden.

\section{Aims \\ To characterise women who died by suicide during pregnancy and postpartum from a maternal care perspective.}

\section{Method}

We traced deaths $(n=103)$ through linkage of the Swedish Cause of Death Register with the Medical Birth and National Patient Registers. We analysed register data and obstetric medical records.

\section{Results}

The maternal suicide ratio was 3.7 per 100000 live births for the period 1980-2007, with small magnitude variation over time. The suicide ratio was higher in women born in low-income countries (odds ratio 3.1 (95\% Cl 1.3-7.7)). Violent suicide methods were common, especially during the first 6 months postpartum. In all, 77 women had received psychiatric care at some point, but 26 women had no documented psychiatric care. Antenatal documentation of psychiatric history was inconsistent. At postpartum discharge, only 20 women had a plan for psychiatric follow-up.

\section{Conclusions}

Suicide prevention calls for increased clinical awareness and cross-disciplinary maternal care approaches to identify and support women at risk.

\section{Declaration of interest}

None.

\section{Copyright and usage}

(c) The Royal College of Psychiatrists 2016.
Sweden has today more than 9 million inhabitants, and about 100000 live births every year. ${ }^{1}$ The World Health Organization (WHO) defines maternal death as the death of a woman while pregnant or within 42 days after termination of pregnancy irrespective of the duration and site of the pregnancy - from any cause related to or aggravated by the pregnancy. Since 2012, suicides occurring during pregnancy and within 42 days after termination of pregnancy are recognised as maternal deaths. ${ }^{2}$ Late maternal deaths are defined as occurring later than 42 days but within 1 year after termination of pregnancy. According to the WHO, the maternal mortality ratio in Sweden (suicides excluded), was 6 per 100000 live births during the years 1988-2007. . $^{3}$

Although the incidence of suicide among women who have given birth during the past 12 months is lower than that of women who have not given birth, ${ }^{4,5}$ in high-income countries suicide remains one of the most common causes of death during the year following delivery. ${ }^{3,6,7}$ The UK Confidential Enquiry into Maternal Deaths (CEMD), which assesses the cause of individual maternal deaths, rates suicide as a leading cause of maternal death in the UK. ${ }^{8}$ They concluded that the suicide rate following delivery generally is similar to that of the general female population, but for a subgroup of women with a serious mental illness, such as severe affective and psychotic disorders, the risk of suicide may be elevated. ${ }^{8}$ Women who die by suicide during or after pregnancy often use violent methods. ${ }^{4,6-8}$ This is in contrast to the preference of women in the non-perinatal period, who usually choose non-violent methods.

The total female suicide rate in Sweden was about 14 per 100000 at the beginning of the 1980s and 9 per 100000 in 2009. ${ }^{9}$ However, although female suicide incidence has decreased, the opposite pattern is found in women aged 15-24 years over the last decade. ${ }^{9}$ Suicide prevention in general is a high public health priority in Sweden. At no other time in their reproductive lifespan do women have more frequent contact with healthcare professionals than during pregnancy and postpartum. Since the objective of maternal healthcare is to identify and reduce risks and ill health, ${ }^{10}$ every suicide during or shortly after pregnancy may represent a lost opportunity for the healthcare system to diagnose and treat psychiatric morbidity.

Sweden today is a multi-ethnic society, and more than every fifth live birth occurs among mothers born abroad. Pregnant immigrant women have been reported as having higher psychiatric morbidity than Swedish pregnant women, ${ }^{11}$ although it has yet to be determined whether or not this translates into a difference in suicide rates. The objective of this study was to describe the population of women in Sweden who died by suicide during their pregnancy or within 1 year after delivery, and to do so from the perspective of maternal care by analysing time trends, psychiatric disorders and treatment, and sociodemographic and obstetric factors.

\section{Method}

The study population consisted of women who died by suicide during pregnancy, or within 1 year after delivery, between 1980 and 2007. We identified them through linkage of the Swedish Cause of Death Register (CDR) with the Medical Birth Register (MBR). We noted all women who died within 1 year after giving birth; ${ }^{3,6}$ we included those with an underlying cause of death due to certain suicide (intentional self-harm, ICD-9: ${ }^{12}$ E950-E959; ICD-10: ${ }^{13}$ X60-X84) or uncertain suicide (undetermined intent, ICD-9: E980-E989; ICD-10: Y10-Y34). From 1988 to 2007, we also cross-referenced the CDR to the National Patient Register 
(NPR), ${ }^{3}$ and thus, we also identified and included women who were recorded as having received specialist care during pregnancy, but who took their own lives before delivery. We excluded 12 women who died by suicide following early miscarriage or extrauterine pregnancy.

We obtained death certificates from the Swedish National Board of Health and Welfare for a total of 103 suicides: 88 were certain suicides, 12 were uncertain and 3 women had a cause of death registered as accidental, although the information on the death certificate and the circumstances surrounding the death suggested probable suicide. We categorised methods of suicide as non-violent if they involved intoxication by drugs or gas. We classified all other methods as violent. We could not obtain 6 medical records, and in the case of 14 additional records, at least one page was missing.

Since our interest in suicide was from a maternal care perspective, we obtained copies of the medical records of the index pregnancy, including delivery and postpartum care, through the respective departments of obstetrics and gynaecology. Three obstetricians scrutinised these records, and noted significant factors with regard to somatic and psychiatric history, sociodemographic situation, complications of pregnancy and delivery, and maternal care. We obtained no medical records from primary care physicians or psychiatric departments. We obtained information on psychiatric drug use from the medical records and categorised this according to the Anatomical Therapeutic Chemical (ATC) groups N06A (antidepressants), N05A (antipsychotics), N05B (anxiolytics) or N05C (hypnotics and sedatives) (www.ephmra.org/Anatomical-Classification). We defined psychiatric care as treatment by a physician, psychologist or social worker, according to the medical record, as specific roles sometimes could not be distinguished.

We obtained data on the women's earlier psychiatric hospital admissions or out-patient contacts with a psychiatric specialist (diagnoses in ICD-9: 290-319; and ICD-10: F00-F99) or suicide attempts (events of intentional self-harm or undetermined intent in ICD-9: E950-E959, E980-E989; and ICD-10: X60-X84, Y10-Y34) from the NPR, and data on the women's pregnancies and perinatal outcomes from the MBR. The NPR has collected data on hospital discharges since 1964, but was not considered complete for all public hospitals until 1987. During the study period, no private maternity or psychiatric in-patient care was available. Beginning in 2001, the NPR has also recorded specialist out-patient care. We obtained sociodemographic data, including country of birth of the background population (consisting of mothers of all live births in Sweden during the study period), from the MBR. The Swedish National Board of Health and Welfare maintains the CDR, MBR and NPR. Countries of birth were listed according to the World Bank country classification of 2007 as low-, middle- and high-income countries, or as Sweden.

We assessed the differences in the distribution of sociodemographic variables between the study population and the background population, and differences in psychiatric morbidity between women who died within the first 6 months versus 6-12 months after delivery with the chi-squared and Fisher's exact tests, with $P$-values $<0.05$ considered statistically significant. We selected a cut-off point at 6 months (182 days) in parallel with the latest report from the UK CEMD. ${ }^{8}$

We calculated odds ratios (ORs) and 95\% confidence intervals (CIs) with the aim to compare suicide ratios for foreign-born and Swedish-born women.

According to Swedish law, ethics approval for this study was not required because all women were deceased. The Regional Ethics Committee in Uppsala, Sweden confirmed that the study did not fall into the category of research requiring ethical clearance (2008/381, 14 January 2009). We asked all heads of the clinical departments where a woman had been cared for to provide consent in sharing a copy of the medical records.

\section{Results}

The maternal suicide ratio was 3.7 per 100000 live births during the entire study period, with small magnitude variation over time (Fig. 1). In Swedish-born women the ratio was 3.5 per 100000 births, for women born in other high-income countries 2.7 $(\mathrm{OR}=0.8,95 \%$ CI $0.3-2.2)$, middle-income countries $5.4(\mathrm{OR}=$ 5.4, 95\% CI 0.9-2.9) and low-income countries $10.8(\mathrm{OR}=3.1$, $95 \%$ CI 1.3-7.7). The maternal mortality ratio, including suicides (within 42 days after termination of pregnancy), was 6.6 per 100000 live births and 6.0 when suicides were excluded. Out of all maternal deaths between 1988 and 2007, 18\% were suicides. In comparison to the background population, the women who died by suicide were significantly more often over 35 years, smokers, not cohabiting and born in low-income countries (Table 1).

Three women died in pregnancy, all of them in the third trimester. Fifty-one deaths occurred within the first 6 months and 49 deaths between 6 and 12 months after delivery (Fig. 2). Violent suicide methods were used by $87 \%$ of women who either died pregnant or within the first 6 months after delivery, whereas the corresponding number was $55 \%$ among women who died 6-12 months postpartum $(P<0.05)$. The suicide methods are shown in Fig. 3. We found no significant differences in sociodemographic factors between the women who died within the first 6 months (day 0-181) and those who died 6-12 months (day 182-364) postpartum (Table 1).

\section{Psychiatric history before pregnancy}

Forty-eight women had a psychiatric history prior to the index pregnancy that was either identified through antenatal records $(n=8)$, the NPR $(n=18)$ or both $(n=22)$ (Table 2$)$. Nearly all women identified by the NPR (39/40) had been admitted to hospital at least once (not shown in Table 2). Seventeen women had sought specialist care due to an injury caused by intentional self-harm (i.e. suicide attempt) between 2 and 17 years before the index pregnancy, but only three of these suicide attempts were documented in the antenatal records (Table 2). Thirteen women had previous or ongoing substance misuse; four identified by the medical records, five by the NPR and four by both sources (Table 2). Experience of violence was reported in three women:

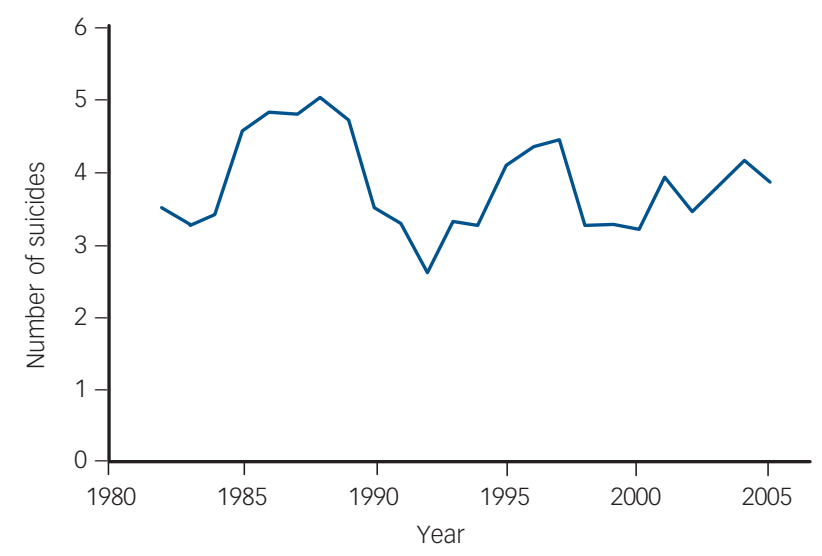

Fig. 1 Number of suicides per 100000 live births (5-year sliding mean values). 


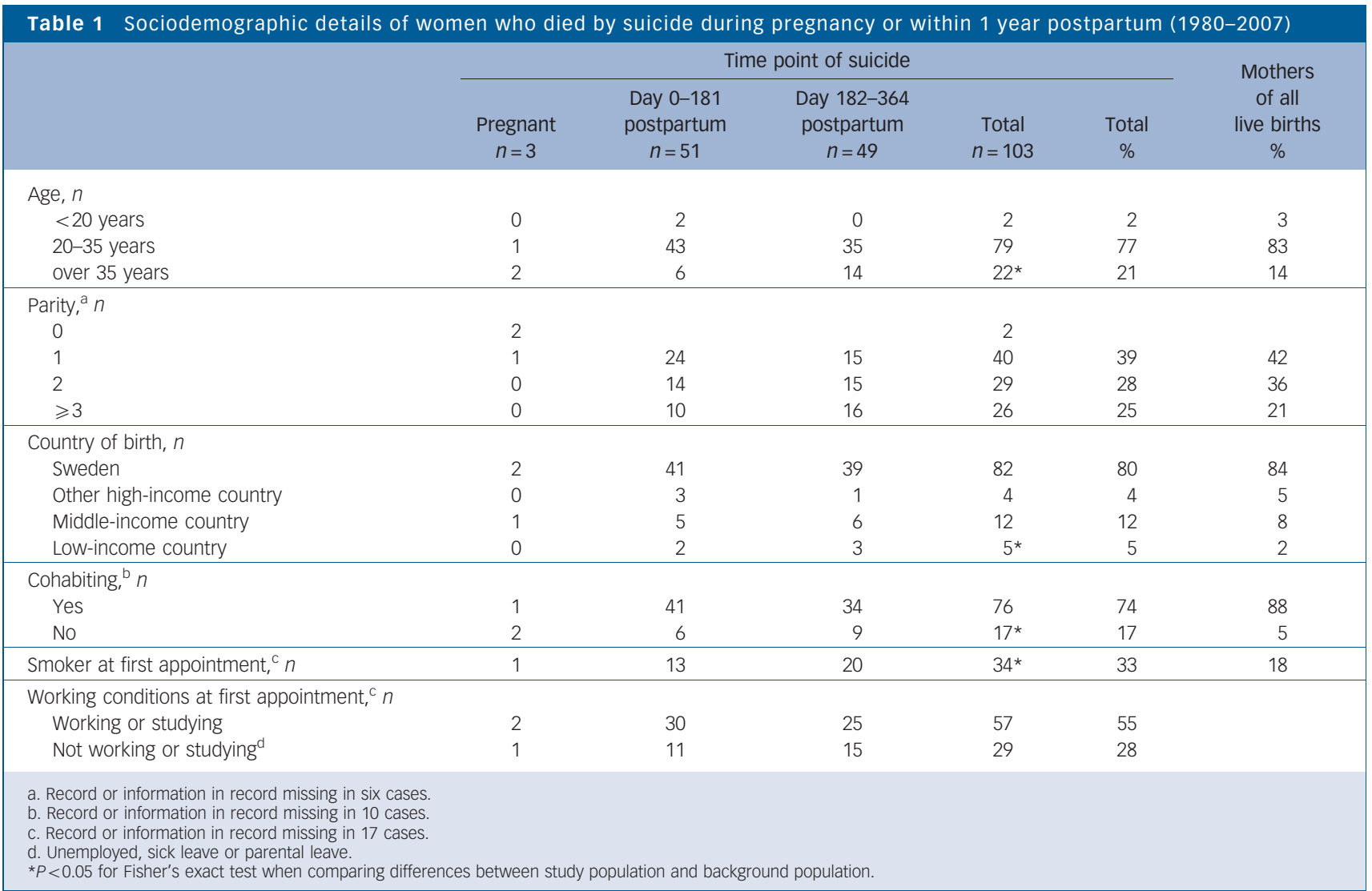

two of them from earlier partners, while the perpetrator was not specified for the third. Women who died by suicide within the first 6 months after delivery had less often had specialist psychiatric contact prior to the index pregnancy, and had less often had a history of suicide attempt than those who took their own lives by suicide $6-12$ months after delivery.

\section{Mental healthcare during pregnancy}

In addition to the 48 women who had a psychiatric history before the pregnancy, 6 women reported an onset of psychiatric symptoms during pregnancy. Of these 54 women, 31 were under

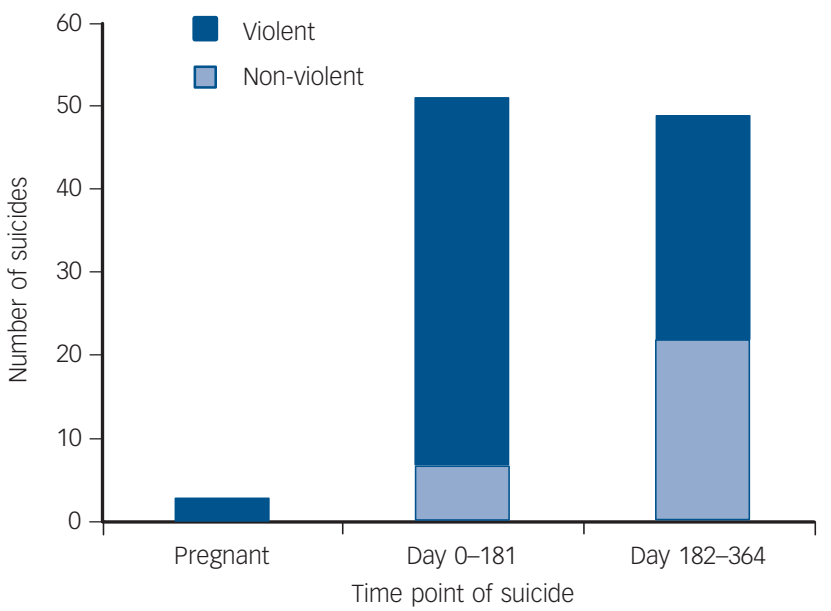

Fig. 2 Time point and method of suicide during pregnancy and within 1 year of delivery in Sweden, 1980-2007. psychiatric care and 8 were admitted to hospital due to a psychiatric disorder during the index pregnancy (Table 3). Three women attempted suicide during the pregnancy, all of whom had a history of suicide attempts.

Nineteen women were treated with one or multiple drugs, including antidepressants, antipsychotics, anxiolytics or hypnotics and sedatives at their first antenatal care appointment or later during pregnancy (Table 3 ). The use of psychoactive drugs was equally common in the early (1980-1993) and latter part (19942007) of the study period. All eleven women taking antidepressant drugs during pregnancy received specialist psychiatric care after delivery. Eight women were treated with selective serotonin reuptake inhibitors (SSRIs), but only three of them throughout the entire pregnancy.

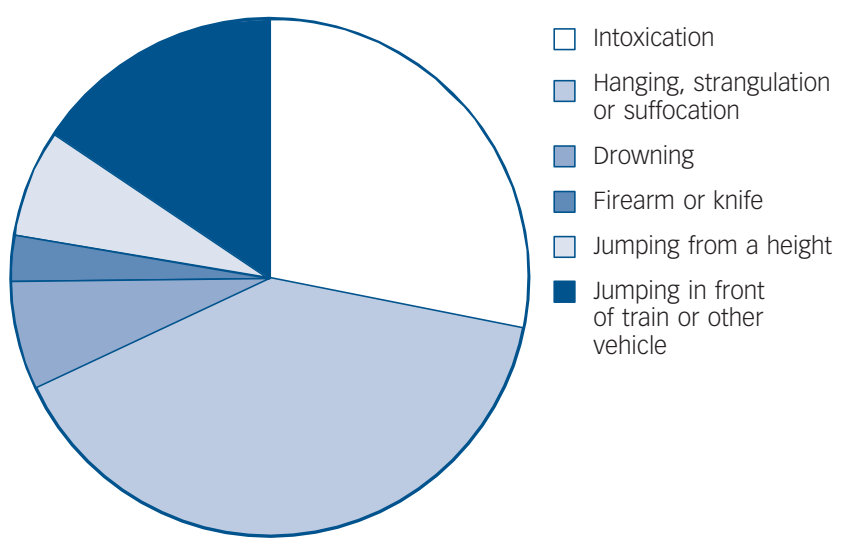

Fig. 3 Methods of suicide during pregnancy and within 1 year after delivery in Sweden, 1980-2007. 


\begin{tabular}{|c|c|c|c|c|}
\hline & \multicolumn{4}{|c|}{ Time point of suicide } \\
\hline & Pregnant & Day 0-181 postpartum & Day 182-364 postpartum & Total \\
\hline Total & $n=3$ & $n=51$ & $n=49$ & $n=103$ \\
\hline Psychiatric disorder according to medical record, ${ }^{a} n$ & 2 & 11 & 17 & $30^{\mathrm{b}}$ \\
\hline Earlier suicide attempt ${ }^{\mathrm{b}}$ & 0 & 2 & 1 & 3 \\
\hline Earlier or ongoing substance misuse ${ }^{c}$ & 0 & 2 & 6 & 8 \\
\hline Psychiatric disorder before pregnancy, according to register, ${ }^{d} n$ & 0 & 12 & $28^{*}$ & $40^{\mathrm{b}}$ \\
\hline Suicide attempt ${ }^{\mathrm{e}}$ & 0 & 4 & $13^{*}$ & 17 \\
\hline Bipolar disorder ${ }^{f}$ & 0 & 2 & 4 & 4 \\
\hline Other mood disorderg & 0 & 2 & 2 & 5 \\
\hline Psychotic disorder ${ }^{\text {h }}$ & 0 & 3 & 5 & 8 \\
\hline Anxiety disorder ${ }^{i}$ & 0 & 4 & $11^{*}$ & 15 \\
\hline Disorder due to psychoactive substance use ${ }^{j}$ & 0 & 1 & $8^{*}$ & 9 \\
\hline Other psychiatric disorderk & 0 & 2 & 9* & 11 \\
\hline \multicolumn{5}{|c|}{$\begin{array}{l}\text { a. Record or information in record missing in eight cases. } \\
\text { b. In total } 48 \text { women had a psychiatric history. Information on psychiatric disorder present in both medical records and register in } 22 \text { cases. } \\
\text { c. Record or information in record missing in } 36 \text { cases. } \\
\text { d. One or more hospital admissions or out-patient specialist appointments due to psychiatric disorder or intentional self-harm recorded in the Swedish National Patient Register prio } \\
\text { to the index pregnancy. } \\
\text { e. Intentional self-harm: ICD-9: E950-E959, E980-E989; ICD-10: X60-X84, Y10-Y34. } \\
\text { f. ICD-9: 296; ICD-10: F31. } \\
\text { g. ICD-9: 311; ICD-10: F30, F32-F39. } \\
\text { h. ICD-9: 295-299; ICD-10: F20-F29. } \\
\text { i. ICD-9: 300; ICD-10: F40-F48. } \\
\text { f. ICD-9: 303-305; ICD-10: F10-F19. } \\
\text { k. Personality, sleep and adjustment disorders. } \\
\text { *P<0.05 difference between women who died by suicide on day 0-181 and day } 182-364 \text { postpartum, chi-squared test. }\end{array}$} \\
\hline
\end{tabular}

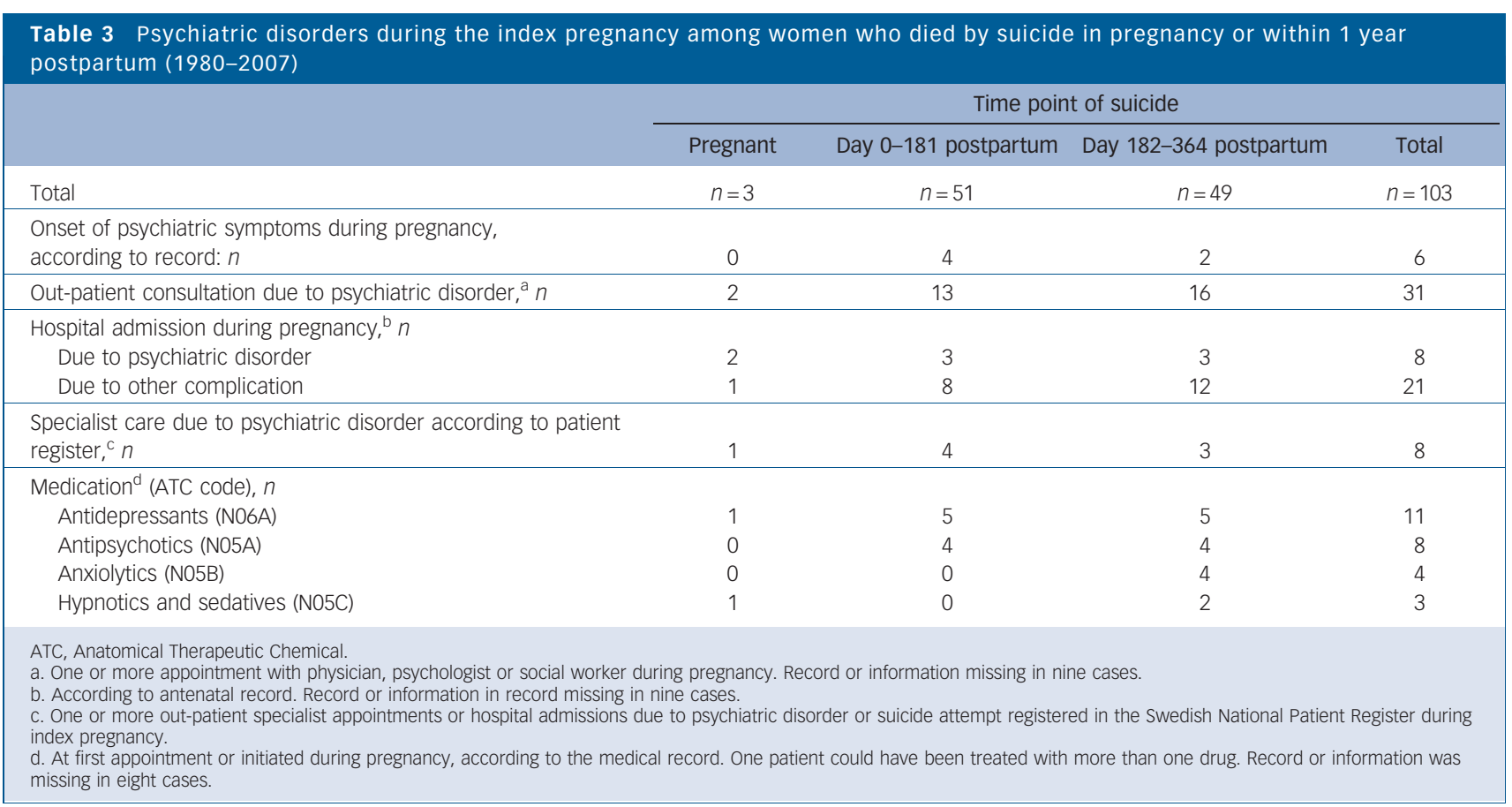

\section{Mental healthcare postpartum}

Among the 54 women who had a documented psychiatric disorder, 21 received psychiatric care soon after delivery according to the medical records. In addition, 6 women had a first onset of psychiatric symptoms in the postpartum period, for which they received psychiatric care. Hence, 27 women received psychiatric care in the immediate postpartum period (Table 4). Only 20 women had a documented plan for psychiatric follow-up at discharge from the maternity ward (Table 4). According to the NPR, 51 women received specialist psychiatric care in the interval between delivery and their death (Table 4). Thirteen of these did not have a documented psychiatric disorder prior to, during or immediately following the pregnancy.
All in all, 77 of the 103 women who died by suicide had been in contact with psychiatric healthcare providers before, during or after the index pregnancy (72 with psychiatric specialist care). Twenty-six women died by suicide without any trace of documentation of psychiatric history or psychiatric care. Nineteen of these took their lives during the first 6 postpartum months, and 17 of the suicides were by violent methods. These women did not differ from the other women with respect to sociodemographic characteristics or perinatal complications.

\section{Perinatal outcome}

Almost half of the women experienced some adverse event during pregnancy or delivery (Table 5). Three women were carrying twin 


\begin{tabular}{|c|c|c|c|}
\hline & \multicolumn{3}{|c|}{ Time point of suicide } \\
\hline & Day 0-181 postpartum & Day $182-364$ postpartum & Total \\
\hline Total & $(n=51)$ & $(n=49)$ & $(n=100)$ \\
\hline \multicolumn{4}{|l|}{ Psychiatric disorder according to medical records, $n$} \\
\hline Onset of psychiatric symptoms & 6 & 0 & 0 \\
\hline Consultation due to psychiatric disorder ${ }^{\mathrm{a}}$ & 18 & 9 & 27 \\
\hline Documented plan for follow-up ${ }^{\mathrm{b}}$ & 13 & 7 & 20 \\
\hline Psychiatric disorder after pregnancy, according to NPR register: ${ }^{c} n$ & 21 & 30 & 51 \\
\hline Suicide attempt ${ }^{d}$ & 4 & 9 & 13 \\
\hline Bipolar disorder ${ }^{\mathrm{e}}$ & 4 & 7 & 11 \\
\hline Other mood disorder ${ }^{f}$ & 5 & 4 & 9 \\
\hline Psychotic disorderg & 8 & 9 & 17 \\
\hline Anxiety disorder ${ }^{\mathrm{h}}$ & 3 & 9 & 12 \\
\hline Disorder due to psychoactive substance use ${ }^{i}$ & 0 & 2 & 2 \\
\hline Other psychiatric disorder ${ }^{j}$ & 2 & 5 & 7 \\
\hline \multicolumn{4}{|c|}{ 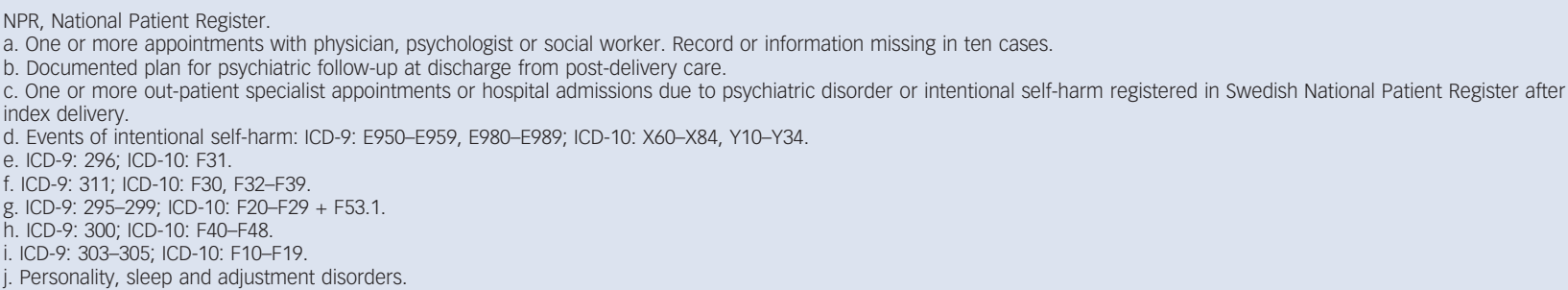 } \\
\hline
\end{tabular}

Table 5 Gestational length, mode of delivery and complications in women who died by suicide during pregnancy or within 1 year postpartum (1980-2007)

\begin{tabular}{|c|c|c|c|c|}
\hline & \multicolumn{4}{|c|}{ Time point of suicide } \\
\hline & Pregnant & Day 0-181 postpartum & Day 182-364 postpartum & Total \\
\hline Total & $n=3$ & $n=51$ & $n=49$ & $n=103$ \\
\hline \multicolumn{5}{|l|}{ First appointment, ${ }^{a} n$} \\
\hline$<18$ gestational weeks & 2 & 44 & 39 & 85 \\
\hline$\geqslant 18$ gestational weeks & 0 & 0 & 5 & 5 \\
\hline \multicolumn{5}{|l|}{ Midwife visits, ${ }^{\mathrm{b}} n$} \\
\hline $3-12$ & 1 & 32 & 36 & 69 \\
\hline$>12$ & 1 & 10 & 8 & 19 \\
\hline \multicolumn{5}{|c|}{ Gestational length at delivery, weeks + days } \\
\hline$<37+0$ & & 3 & 5 & 8 \\
\hline $37+0$ to $41+6$ & & 44 & 43 & 87 \\
\hline$\geqslant 42+0$ & & 4 & 1 & 5 \\
\hline \multicolumn{5}{|l|}{ Mode of delivery, $n$} \\
\hline Unassisted vaginal & & $43^{c}$ & $38^{d}$ & $81^{c, d}$ \\
\hline vacuum extraction & & $4^{c}$ & 2 & $6^{\mathrm{C}}$ \\
\hline Elective caesarean & & 1 & 4 & 5 \\
\hline Emergency caesarean & & 4 & $5^{e}$ & $9^{e, f}$ \\
\hline Adverse events during pregnancy, ${ }^{g} n$ & 2 & 22 & 22 & 46 \\
\hline Adverse events during delivery, $^{\mathrm{h}} n$ & & 27 & 21 & 48 \\
\hline \multicolumn{5}{|c|}{$\begin{array}{l}\text { a. Record or information missing in } 13 \text { cases. } \\
\text { b. Record or information missing in } 15 \text { cases. } \\
\text { c. One twin delivery with one vaginal and one vacuum extraction. } \\
\text { d. One premature twin delivery, gestational week } 29 \text {. } \\
\text { e. One twin delivery. } \\
\text { f. One woman arrived at hospital as a multitrauma case and underwent perimortem caesarean section at admission, but infant did not survive. She is not included in the table. } \\
\text { g. Hypertensive disorder, pre-eclampsia, intrauterine fetal death, intrauterine growth retardation, diabetes, placenta praevia, preterm premature rupture of membranes, vaginal } \\
\text { bleeding, duplex, premature contractions or pelvic pain requiring hospital admission or sick leave, post-term pregnancy, restless legs, polyhydramnios, myoma, genital herpes, } \\
\text { thyroid disorder. Record or information missing in eight cases. } \\
\text { h. Induced labour, preterm labour, instrumental delivery, fetal distress, dystocia, retained placenta, postpartum haemorrhage. }\end{array}$} \\
\hline
\end{tabular}

pregnancies, two pairs delivered at full term and one pair was born at 29 gestational weeks. Another seven deliveries were preterm ( $>35$ but $<37$ gestational weeks).

The 100 pregnancies of mothers who died by suicide after delivery resulted in 102 live births. Nineteen infants suffered neonatal complications other than prematurity, such as asphyxia, infection, small for gestational age, neonatal respiratory distress, hypoglycaemia, fractured clavicle, cephalohematoma or congenital malformation (including one baby with a ventricular septum defect, four babies with musculoskeletal abnormalities and one with hypospadias). One woman had an intrauterine fetal death and one child died within 30 days of birth. There were two cases of suicide and infanticide. 


\section{Foreign-born women}

Of the 21 women who were born abroad, 4 came from highincome Nordic countries, 12 from middle-income countries (Poland, Russia, Turkey, the former Yugoslavia, Lebanon, Iraq, China, Chile and Botswana) and 5 from low-income countries (Ethiopia, Somalia, India and Bangladesh). One woman was an international adoptee who had come to Sweden in early childhood. In the majority of cases, information on how long they had lived in Sweden was not available. Twelve women had a psychiatric history prior to pregnancy; one had a first onset during pregnancy and two during the postpartum period. The remaining six women had no documentation of psychiatric disorder in their clinical records or the NPR. Documented language barriers were present in four cases, although a professional interpreter was only used in one.

\section{Discussion}

\section{Major findings}

The ratio of maternal suicides displayed only small variations in magnitude over the study period, and the risk for women born in low-income countries was higher than for Swedish-born women. In parallel with other studies, the majority of women who died by suicide during and after pregnancy had received specialist psychiatric care, and we found psychiatric morbidity prior to pregnancy to be common. ${ }^{7,8}$ Nevertheless, in a fourth of the women no documentation of a psychiatric disorder was found. The common use of violent methods in postpartum suicide has been noted earlier. ${ }^{4,6-8}$ However, here we report on important differences in both pre-pregnancy psychiatric morbidity and method of suicide between women who died by suicide within 6 months and from 6-12 months of delivery. Women who took their own lives during the first 6 months postpartum less often had a psychiatric history and more often used violent methods of suicide, suggesting different risk profiles.

\section{Psychiatric morbidity among the women who died by suicide}

Suicides are almost always associated with mental illness. This was also the case in the present study, where more than $75 \%$ of women had psychiatric contact before, during or after the index pregnancy. ${ }^{14}$ The estimated prevalence of major depressive disorder is approximately $7 \%$ during pregnancy and $6 \%$ during the first 3 months postpartum, ${ }^{15}$ which is equivalent to the $6.3-10.3 \%$ 1-year prevalence found in general population surveys. ${ }^{16}$ Anxiety disorders are less studied, but their prevalence seem to be high during both pregnancy $(11 \%)$ and the postpartum period $(8 \%),{ }^{15}$ which is equivalent to the $5.6-18.1 \%$ 1 -year prevalence in the general population. ${ }^{16}$ The overall risk of developing a psychotic episode is increased during the year after childbirth, ${ }^{17}$ and 1-2/1000 women giving birth are admitted to hospital due to puerperal psychosis. ${ }^{18}$ Women with bipolar disorder constitute a particular challenge, as the risk for manic/ depressive or psychotic episodes is substantially elevated postpartum. ${ }^{19}$ Almost $20 \%$ of women with bipolar disorder are admitted to hospital within the first months postpartum. ${ }^{20}$ Prior psychiatric hospital admission is a strong risk factor for postpartum psychiatric admission ${ }^{20}$ and suicide attempts. ${ }^{21}$

Close contacts with the healthcare system during pregnancy and the postpartum period provide prime opportunities for detecting and treating serious psychiatric disorders. ${ }^{10}$ Additionally, the UK CEMD have repeatedly recommended that women should be interviewed about psychiatric history and current mental health problems at their first antenatal visit. ${ }^{8}$ In Sweden, this procedure was routine during the study period; however, only 3 of 17 cases of suicide attempts, and 22 of 40 cases with previous specialist psychiatric care were documented at the first antenatal appointment. The documentation of psychiatric history in the antenatal records was often short and imprecise, and did not enable classification of a disorder. Furthermore, as a suicide attempt remains a lifelong major risk factor for suicide, ${ }^{14}$ women who report a psychiatric history at their first antenatal appointment should also be questioned about any previous suicide attempts. Experience of violence is also a risk factor for psychiatric disorders ${ }^{22}$ and suicide, ${ }^{23}$ although in our population it was only documented in three antenatal records. Experiences of adverse events during pregnancy and delivery were common; almost half had complications during pregnancy, and almost half had complicated deliveries. In addition, 19 children had some type of problem in the neonatal period. Associations between perinatal complications and postpartum depression in otherwise healthy women have been demonstrated in earlier studies, ${ }^{24,25}$ and in this vulnerable group of women, the added adversity of pregnancy, delivery or child complications may have been detrimental.

Despite the well-known risk of postpartum deterioration of several psychiatric disorders, ${ }^{8,17,19,21}$ a documented psychiatric follow-up plan following discharge from the maternity ward was only present in 20 out of the 31 women who had received psychiatric care during pregnancy. Nevertheless, 51 women had specialist psychiatric contacts in the time interval between delivery and death. The 26 women who had no documentation of psychiatric disorders are of particular interest. We can only speculate what their circumstances might have been. It is possible that they had psychiatric symptoms that were not correctly interpreted, or that the onset was very rapid. In addition, they may have sought psychiatric out-patient care before 2001, prior to records of such care began being kept.

It has been standard practice in Sweden during the study period to separate mother and child should the mother need admission to a psychiatric ward. Unfortunately, this information was not available for our study. There is little knowledge as to how the separation from the newborn would influence the mother's psychiatric disorder. However, what is known is that active support of mentally ill mothers improves the interaction between mother and child, which in turn, is essential for the child's development. ${ }^{26}$ The UK CEMD therefore strongly recommend mother and baby units for all women requiring psychiatric admission in late pregnancy and the postpartum period so that the women are not separated from their infants. ${ }^{8}$ To the best of our knowledge, such units do not presently exist in Sweden.

\section{Suicides among foreign-born women}

For women born in low-income countries, the risk of suicide during pregnancy and postpartum was elevated. Non-native, Swedish-speaking pregnant women have higher psychiatric morbidity, yet they are less likely to seek psychiatric care. ${ }^{11}$ This may be specific to Sweden. A Danish study has shown that psychiatric contacts were more common among foreign-born women (irrespective of origin) than among native Danes during pregnancy and the year postpartum. ${ }^{27}$ Region of origin was also found to be a strong predictor for antenatal depressive symptoms in a Canadian study: women from South Asia and sub-Saharan Africa were among those with the greatest prevalence of depressed mood. ${ }^{28}$ A language barrier was documented in the case of four women in our study, and yet a professional interpreter was used in only one instance, thereby underscoring the necessity to use professional medical interpreters for all non-native-speaking women. ${ }^{8}$ 


\section{Similarities and differences with the findings from the UK CEMD}

The similarities between this study and the UK CEMD findings are compelling. Both show the importance of a history of psychiatric ill health, as well as the older age of the suicide victims, and the frequent use of violent methods of death. ${ }^{8}$ Similarly, both studies indicate that the relative numbers of women dying due to maternal suicide has not changed over time. However, differences between the two settings are also at hand. The maternal suicide rate in Sweden was higher than in the UK. In Sweden, there was also a higher proportion of women who had received psychiatric care before and during pregnancy, and a lower proportion of women who had postpartum-onset conditions. A difference in sociodemographic characteristics is also noted, in particular the relatively large proportion of foreign-born women in Sweden.

\section{Strengths and limitations}

Our population-based study is strengthened by the fact that all suicides were identified from records held in Swedish national health registers. We were able to access all but six records, and information was complemented by register data. One limitation was the small sample size due to the near rarity of suicide in a small population. Since we identified only three suicides during pregnancy, this study mainly contributes to knowledge on suicide in the postpartum period. Data collection of psychiatric care included only diagnoses and whether out- or in-patient care was provided. Hence, analysis of the association between pregnancy and the possible deterioration of a psychiatric disorder in an individual case was not feasible. Furthermore, we had no information about psychiatric care prior to the suicide, i.e. medication use or electroconvulsive therapy for severe depression or psychosis, and whether care was compulsory or on a voluntary basis. Access to psychiatric records could have enabled the audit of the psychiatric care, but was beyond the scope of this study.

The extended study period meant that some major changes in Swedish psychiatric care have occurred, but our sample was too small to be adjusted for calendar time. The number of psychiatric admissions have decreased dramatically and out-patient care has increased, but the provision of specialised psychiatry has not extended equally to all regions, which could be disadvantageous for some women. ${ }^{29}$ In addition, tricyclic antidepressants have been replaced by SSRIs, which has seen increasing use among women, although less so during pregnancy than postpartum. ${ }^{30}$ In our study, eight women used SSRIs during pregnancy, but only three during their entire pregnancy. Finally, the last decade of the study period included the introduction of screening with the Edinburgh Postnatal Depression Scale for major depression at 6-8 weeks postpartum, ${ }^{31}$ which has increased the overall awareness of postpartum mental ill health.

By including uncertain suicides we may have included some accidental deaths, but we assessed this risk of overestimation to be lower than the risk of underestimating the true number of suicides by excluding all uncertain suicides. Additionally, because the NPR was not complete for hospital admissions before 1987 and registration of out-patient specialist care did not begin until 2001, an underestimation of women having had contacts with psychiatric care may have occurred.

\section{Implications for clinical practice}

Overall, in Sweden suicides during pregnancy and the year following delivery have not decreased, and women born in low-income countries are at highest risk. Among the women who die by suicide, psychiatric morbidity prior to pregnancy is common, although we noted discrepancies between actual and documented psychiatric history. As repeatedly recommended by the UK CEMD, all women should be asked at their first antenatal appointment about a history of psychiatric disorder as well as their current mental health. Pregnant women with a history of severe affective disorder or psychoses should be referred for psychiatric assessment and management, even if they are well. A minimum requirement for management should be regular monitoring and support for at least 3 months following delivery. ${ }^{8}$ Of note, this study shows that obstetric healthcare providers may not understand the importance of previous serious psychiatric disorders. A management plan for the postpartum period was generally found lacking, despite known psychiatric disorders or symptoms. Collaboration between psychiatric and obstetric services is therefore crucial to optimise care and reduce the risk for suicide among women during pregnancy and the year after delivery.

Annika Esscher, MD, PhD, Birgitta Essén, MD, PhD, Department of Women's and Children's Health, International Maternal and Child Health (IMCH), Uppsala University Hospital, Uppsala; Eva Innala, MD, PhD, Department of Clinical Sciences, Obstetrics and Gynaecology, Umeå University, Umeå; Fotios C. Papadopoulos, MD, PhD, Department of Neuroscience, Psychiatry, Uppsala University Hospital, Uppsala; Alkistis Skalkidou, MD, PhD, Inger Sundström-Poromaa, MD, PhD, Ulf Högberg, $\mathrm{PhD}, \mathrm{MD}$, Department of Women's and Children's Health, Uppsala University Hospital, Uppsala, Sweden

Correspondence: Annika Esscher, Department of Women's and Children's Health (IMCH), Uppsala University, Akademiska sjukhuset, SE-751 85 Uppsala, sweden; Email: annika.esscher@kbh.uu.se

First received 9 Jun 2014, final revision 10 Dec 2014, accepted 5 Feb 2015

\section{Funding}

This work was supported by a grant from the Swedish Council for Working Life and Social Research [FAS 2007-2026] and by the Medical Faculty of Uppsala University.

\section{Acknowledgements}

We thank Emelie Hallberg for assisting in collating medical records for the 1988-2007 period.

\section{References}

1 Statistics Sweden. Population Database. Statistics Sweden, 2014.

2 World Health Organization. The WHO Application of ICD-10 to Deaths During Pregnancy, Childbirth and the Puerperium: ICD-MM. WHO, 2012.

3 Esscher A, Högberg U, Haglund B, Essén B. Maternal mortality in Sweden 1988-2007: more deaths than officially reported. Acta Obstet Gynecol Scand 2013; 92: 40-6

4 Appleby L. Suicide during pregnancy and in the first postnatal year. $B M$ 1991; 302: 137-40.

5 Gissler M, Berg C, Bouvier-Colle MH, Buekens P. Injury deaths, suicides and homicides associated with pregnancy, Finland 1987-2000. Eur J Public Health 2005; 15: 459-63.

6 Högberg U, Innala E, Sandström A. Maternal mortality in Sweden, 1980-1988. Obstet Gynecol 1994; 84: 240-4.

7 Austin MP, Kildea S, Sullivan E. Maternal mortality and psychiatric morbidity in the perinatal period: challenges and opportunities for prevention in the Australian setting. Med J Aus 2007; 186: 364-7.

8 Cantwell R, Clutton-Brock T, Cooper G, Dawson A, Drife J, Garrod D, et al. Saving mothers' lives: Reviewing maternal deaths to make motherhood safer, 2006-2008. The Eighth Report of the Confidential Enquiries into Maternal Deaths in the United Kingdom. BJOG 2011; 118(Suppl 1): 1-203.

9 Titelman D, Oskarsson H, Wahlbeck K, Nordentoft M, Mehlum L, Jiang GX, et al. Suicide mortality trends in the Nordic countries 1980-2009. Nord J Psychiatry 2013; 67: 414-23.

10 Swedish Society of Obstetrics and Gynecology. Mödrahälsovård, Sexuell Och Reproduktiv Hälsa [Antenatal Care, Sexual and Reproductive Health]. SFOG, 2008 
11 Wangel AM, Schei B, Ryding EL, Östman M, Bidens Study Group. Mental health status in pregnancy among native and non-native Swedish-speaking women: a Bidens study. Acta Obstet Gynecol Scand 2012; 91: 1395-401.

12 World Health Organization. ICD-9: International Statistical Classification of Diseases (9th Revision). WHO, 1975.

13 World Health Organization. ICD-10: International Statistical Classification of Diseases and Related Health Problems (10th Revision). WHO, 2007.

14 Hawton K, van Heeringen K. Suicide. Lancet 2009; 373: 1372-81.

15 Andersson L, Sundström-Poromaa I, Wulff M, Åström M, Bixo M. Depression and anxiety during pregnancy and six months postpartum: a follow-up study. Acta Obstet Gynecol Scand 2006; 85: 937-44.

16 Baumeister $\mathrm{H}$, Härter $\mathrm{M}$. Prevalence of mental disorders based on general population surveys. Soc Psychiatry Psychiatr Epidemiol 2007; 42: 537-46.

17 Terp IM, Mortensen PB. Post-partum psychoses. Clinical diagnoses and relative risk of admission after parturition. Br J Psychiatry 1998; 172: 521-6.

18 Valdimarsdóttir U, Hultman CM, Harlow B, Cnattingius S, Sparén P. Psychotic illness in first-time mothers with no previous psychiatric hospitalizations: a population-based study. PLOS Med 2009; 6: e13.

19 Marangell LB. Current issues: women and bipolar disorder. Dialogues Clin Neurosci 2008; 10: 229-38.

20 Munk-Olsen T, Laursen TM, Mendelson T, Pedersen CB, Mors O, Mortensen PB. Risks and predictors of readmission for a mental disorder during the postpartum period. Arch Gen Psychiatry 2009; 66: 189-95.

21 Comtois KA, Schiff MA, Grossman DC. Psychiatric risk factors associated with postpartum suicide attempt in Washington State, 1992-2001. Am J Obstet Gynecol 2008; 199: 120 e1-5.

22 Howard LM, Oram S, Galley H, Trevillion K, Feder G. Domestic violence and perinatal mental disorders: a systematic review and meta-analysis. PLoS Med 2013; 10: e1001452.
23 Palladino CL, Singh V, Campbell J, Flynn H, Gold KJ. Homicide and suicide during the perinatal period: findings from the National Violent Death Reporting System. Obstet Gynecol 2011; 118: 1056-63.

24 Verdoux H, Sutter AL, Glatigny-Dallay E, Minisini A. Obstetrical complications and the development of postpartum depressive symptoms: a prospective survey of the MATQUID cohort. Acta Psychiatr Scand 2002; 106: 212-9.

25 Blom EA, Jansen PW, Verhulst FC, Hofman A, Raat H, Jaddoe VW, et al. Perinatal complications increase the risk of postpartum depression. The Generation R Study. BJOG 2010; 117: 1390-8.

26 Kenny M, Conroy S, Pariante CM, Seneviratne G, Pawlby S. Mother-infant interaction in mother and baby unit patients: before and after treatment. I Psychiatr Res 2013; 47: 1192-8.

27 Munk-Olsen T, Laursen TM, Mendelson T, Pedersen CB. Perinatal mental disorders in native Danes and immigrant women. Arch Womens Ment Health 2010; 13: 319-26.

28 Miszkurka M, Goulet L, Zunzunegui MV. Contributions of immigration to depressive symptoms among pregnant women in Canada. Can J Public Health 2010; 101: 358-64.

29 Tiainen A, Edman G, Flyckt L, Tomson G, Rehnberg C. Regional variations and determinants of direct psychiatric costs in Sweden. Scand J Public Health 2008; 36: 483-92.

30 Munk-Olsen T, Gasse C, Laursen TM. Prevalence of antidepressant use and contacts with psychiatrists and psychologists in pregnant and postpartum women. Acta Psychiatr Scand 2012; 125: 318-24.

31 Wickberg B, Hwang CP. The Edinburgh Postnatal Depression Scale: validation on a Swedish community sample. Acta Psychiatr Scand 1996; 94: 181-4. 